TAO, Vol. 17, No. 2, 353-370, June 2006

\title{
Eustatic Sea-Level Change of 11 - 5 ka in Western Taiwan, Constrained by Radiocarbon Dates of Core Sediments
}

\author{
Meng-Long Hsieh ${ }^{1, *}$, Tzu-Hua Lai ${ }^{2}$, Leh-Chun Wu ${ }^{3}$, Wan-Chung Lu ${ }^{2}$, \\ Hsien-The Liu ${ }^{2}$ and Ping-Mei Liew ${ }^{1}$
}

(Manuscript received 15 January 2005, in final form 16 January 2006)

\begin{abstract}
Twenty-nine cores and two outcrops in the Western Coastal Plain, each containing more than one radiocarbon date ( 5 - $20 \mathrm{ka}$; totally 74 dates), are used to determine the 11 - 5 ka eustatic sea level in Taiwan. All the dates are obtained from coastal sediments with deposition positions assumed $\pm \mathbf{3} \mathbf{~ m}$ of the sea level. These dates can be used to calculate rock uplift (subsidence) rates for given eustatic sea-level data. The sea level data derived from Penghu ( $<5 \mathrm{ka}$ ) and the Sunda Shelf (> $11 \mathrm{ka}$ ) are applied, and the rock uplift of the study sites is assumed to be steady over time. The 11 - $5 \mathrm{ka}$ sea level is constructed by repetition of changing its shape until the dates in the same cores yield consistent rock-uplift rates. Optimal sea-level curves are found to fit all but seven dates used. These sea-level curves are characterized by: (1) a rapid rise (> $13 \mathrm{~m} \mathrm{ky}^{-1}$ ) around $11-10 \mathrm{ka}$; (2) a rise of $8-9 \mathrm{~m} \mathrm{ky}^{-1}$ from $\sim 10 \mathrm{ka}$ to 6.5 - $6.6 \mathrm{ka}$ when the sea level approached the modern level, and (3) a much slower rise after 6.5 - $6.6 \mathrm{ka}$.
\end{abstract}

(Key words: Core, Eustatic sea level, Rock uplift)

\section{INTRODUCTION}

The mountain belt of Taiwan is bounded to the southwest by the Western Coastal Plain (Fig. 1). The Central Geological Survey has drilled over 100 cores in this coastal plain in the

\footnotetext{
${ }^{1}$ Department of Geosciences, National Taiwan University, Taipei, Taiwan, ROC

2 Central Geological Survey, Ministry of Economic Affairs, Taiwan, ROC

${ }^{3}$ Department of Geology, Chinese Culture University, Taipei, Taiwan, ROC

* Corresponding author address: Prof. Meng-Long Hsieh, Department of Geosciences, National Taiwan University, Taipei, Taiwan, ROC; E-mail: hsiehml@ntu.edu.tw
} 


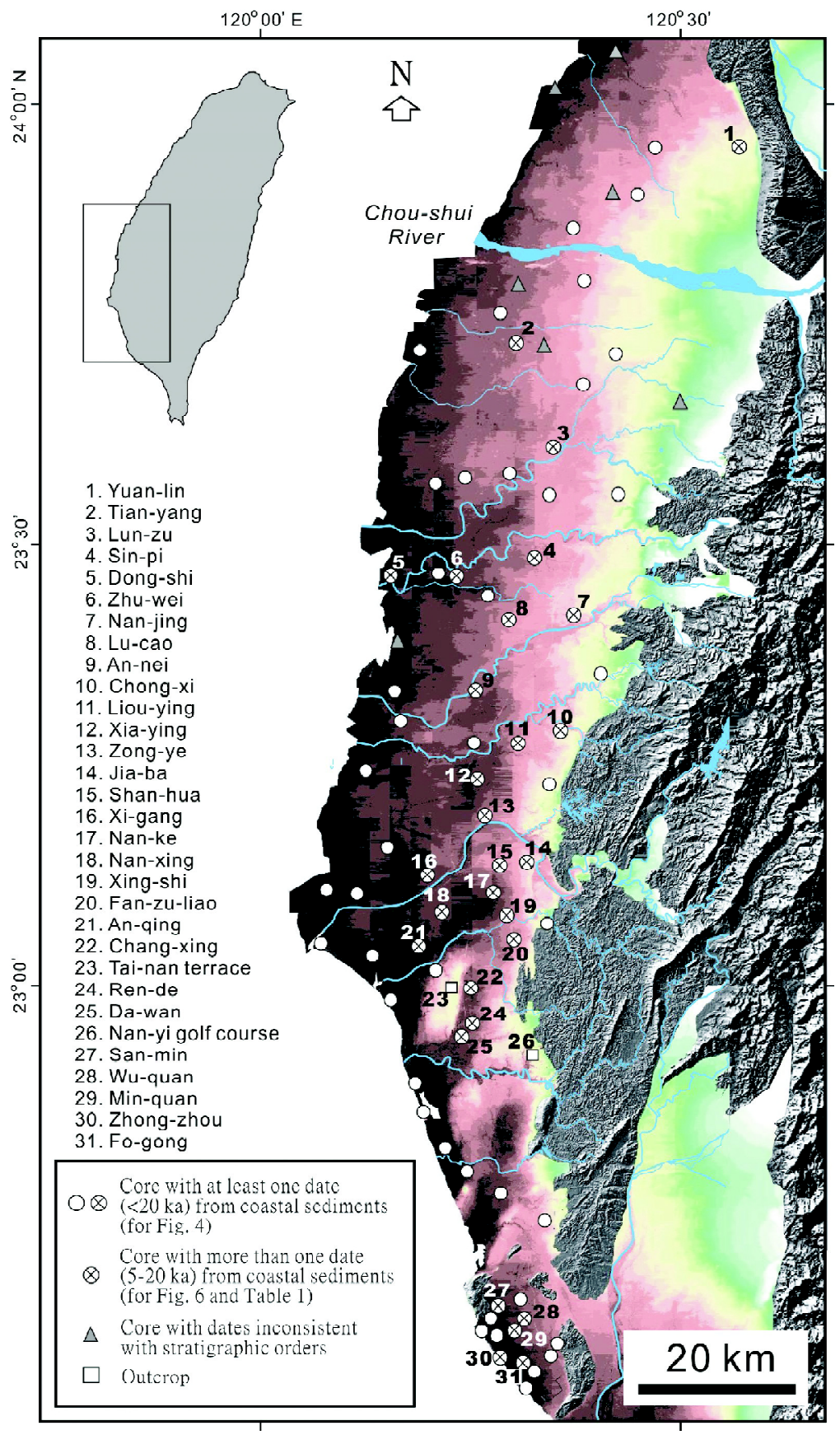

Fig. 1. Location of cores and outcrops with radiocarbon dates examined in this study. The data from sites 23, 25, and 26 by Chen and Liu (2000); others from the Central Geological Survey, ROC. 
past decade to monitor groundwater activity (Central Geological Survey 1999, 2001; Su et al. 1999; Hu et al. 2001). These cores have been logged, with sedimentary sequences interpreted, yielding over 200 radiocarbon dates, mostly younger than 20 ka (e.g., Lai 1995; Huang 2001; Song 2001; Wu 1999, 2004). These data facilitate exploring the region's relative sea-level history since the last glacial maximum.

The paleo-sea-level position for given cores is given by the present elevation $(E)$ of the core sediments corrected by their deposition position $(D)$ relative to the coeval sea level (negative indicating below), as follows:

$$
S_{\text {rel }}=E-D \text {, }
$$

where $S_{\text {rel }}$ denotes the (relative) sea-level position when the sediments were deposited. Being far away from the continental ice sheets and characterized by active tectonics, the sea level in Taiwan is mainly the sum of eustatic and tectonic factors, with certain degrees of sediment compaction, or:

$$
S_{\text {rel }}=S_{\text {eu }}+U \times A-C,
$$

where $S_{\text {eu }}$ denotes the eustatic sea level at time $A$ (relative to the modern sea level; negative indicating below); $U$ denotes rock uplift rate (negative for subsidence), and $C$ is the amount of subsidence due to sediment compaction. In Eqs. 1 and 2, $A$ can be determined by dating the core sediments; $D$ can be inferred from depositional features of the sediments, and $C$ may be negligible for deeper/older sediments, leaving $S_{\text {eu }}$ and $U$ as two major unknowns. The uncertainties in determining these two unknowns in Taiwan are expected to exceed the effects of other minor factors, such as glacial- and hydro-isostasy, on the sea-level history. For simplicity, the term eustatic sea level was used in this study to denote the sea level derived by removing the effects of tectonics and sediment compaction.

The eustatic sea level in Taiwan in the past 5 ky has been established by Chen and Liu (1996) by dating coral platforms in a presumably tectonically stable island, Penghu Island, off western Taiwan (Fig. 2). No eustatic sea-level records before 5 ka have been compiled based on data in or near Taiwan. Taiwan's eustatic sea level before $11 \mathrm{ka}$ can be assumed to be similar to that obtained from the Sunda Shelf (Indonesia) (Hanebuth et al. 2000) (Fig. 2). The eustatic sea level between 11 and $5 \mathrm{ka}$ in Taiwan is less certain. Sea-level records in this period reported around the Pacific vary considerably (Chen and Liu 2000, and references therein), and are constructed from less reliable data than the Sunda Shelf. Chen and Liu (2000) nonetheless smoothed and averaged all these records, and used a straight line, $-2 \mathrm{~m}$ at $6 \mathrm{ka}$ and $-30 \mathrm{~m}$ at $10 \mathrm{ka}$, to approximate the sea level from 10 to $6 \mathrm{ka}$ (Fig. 2).

This study used the core data reported from the Western Coastal Plain to refine the 11 $5 \mathrm{ka}$ eustatic sea level in Taiwan. The eustatic sea level in Taiwan before $11 \mathrm{ka}$ was assumed to be the same as the sea level observed at the Sunda Shelf. Also, the rock uplift of the core sites was assumed to be steady over time (although it may vary among sites). The cores that comprise more than one date derived from coastal sediments were chosen. For given eustatic sea- 


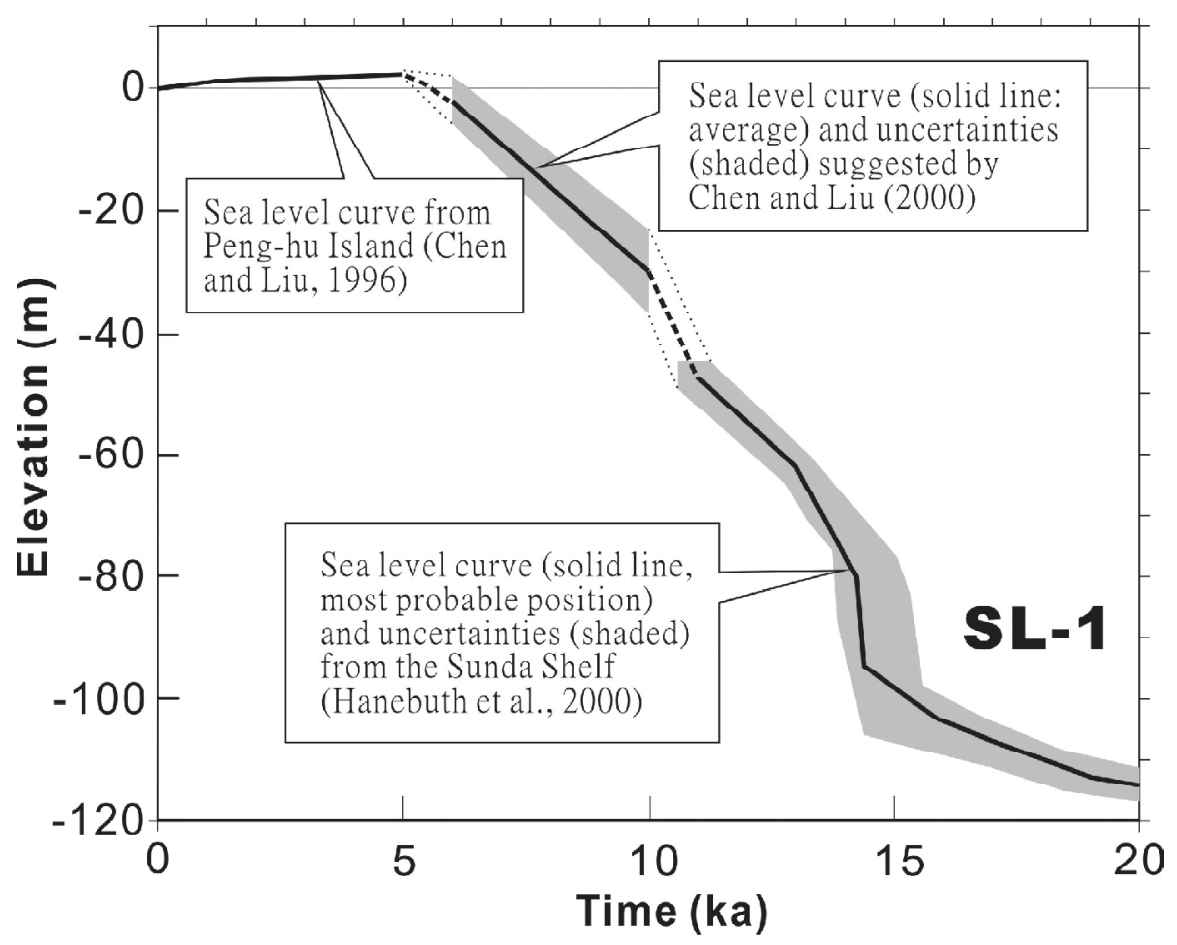

Fig. 2. Sea level SL-1 (and its uncertainty) referred in this study. Sea-level curves during 5 - $6 \mathrm{ka}$ and $10-11 \mathrm{ka}$ (dashed) and uncertainty ranges (dotted) are assumed.

level data, these dates from different elevations produced more than one rock-uplift rate for each of the cores, which can be formulated, based on Eqs. 1 and 2, as:

$$
U=\frac{E-S_{\mathrm{eu}}-D+C}{A} .
$$

An 11 - 5 ka eustatic sea-level curve (or curves) was then found by repetition of changing the curve's shape until the dates obtained from the same cores yielded consistent rock-uplift rates. The feasibility of this method depends on the applicability of the assumptions of steady rock uplift and negligible sediment compaction, and on the reliability of the dates (i.e., if the assumptions are inappropriate or if most of the dates are erroneous, then the obtained sea-level curve can only fit a small part of the data reported). This study tested the data for sediment compaction, and eliminated the younger dates $(<5 \mathrm{ka})$ derived from the sediments that show persistent compaction. Excluding these dates, the proposed 11 - $5 \mathrm{ka}$ eustatic sea-level curve fits most of the available data, implying that most of the reported dates can be considered reliable, and the obtained sea-level curve (and the assumption) can be considered reasonable. 


\section{PROCEDURES}

\subsection{Data Sorting}

All the radiocarbon dates $(<20 \mathrm{ka})$ available for the Western Coastal Plain were examined. All the dates were calibrated using the Radiocarbon Calibration Program (CALIB REV4.4.2) (Stuiver and Meimer 1993). Seven cores contained dates that were inconsistent with their stratigraphic orders (Fig. 1), and were excluded from this study, because the problematic dates within these cores could not be eliminated.

This study focused on the dated sediments showing features that imply deposition near sea level. These sediments were located in the sequence between weathered fluvial strata and shell-bearing marine strata (Lai 1995, Huang 2001, Song 2001, and Wu 1999, 2004 include full interpretation criteria). Coastal sediments that were rich in organic (carbon) material, plant fragments (charcoal), or in-situ roots were selected, as they are likely to have been deposited with coastal vegetation, which generally grows no lower than low-tide level. Where these carbon-rich sediments are associated with marine shells, they are likely to have been deposited under the mean sea level, such as in a lagoon or a tidal-flat setting. Where these sediments do not contain marine shells, and show slight weathering features, they were probably deposited above the mean sea level, such as in a coastal marsh. Figure 3 shows examples of these sediments. The regional tidal range is generally less than $2 \mathrm{~m}$. However, uncertainty ranges of 0 to $-3 \mathrm{~m}$ for the deposition position of the inferred lagoon/tidal-flat sediments, and of 3 to $0 \mathrm{~m}$ for the coastal-marsh sediments, were assigned to incorporate any subjectivity (e.g., the carbon abundance) and uncertainties in determining the deposition settings. An uncertainty range of 3 to $-3 \mathrm{~m}$ was assigned for the deposition position of some carbon-rich coastal sediments without diagnostic marine/fluvial features (i.e., with neither marine shells nor weathering features).

Totally 127 dates $(<20 \mathrm{ka}$ ) from 70 cores (Fig. 1) were obtained from the coastal sediments specified above. These dates were used to calculate rock-uplift rates, according to Eq. 3, by first applying the eustatic sea-level data compiled by Chen and Liu $(1996,2000)$ and Hanebuth et al. (2000) (hereafter Sea level SL-1; Fig. 2). The effects of sediment compaction were temporarily ignored (assuming $C=0$ ), and the $1 \sigma$ ranges of the calibrated ages were assigned for the uncertainties of the dates. These factors, together with the assumed uncertainties for the deposition position, were used to calculate possible ranges of rock-uplift rates:

$$
U_{L}=\frac{E-S_{\mathrm{eu} U}-D_{U}}{A_{L}}
$$

and

$$
U_{U}=\frac{E-S_{\mathrm{eu} L}-D_{L}}{A_{U}},
$$




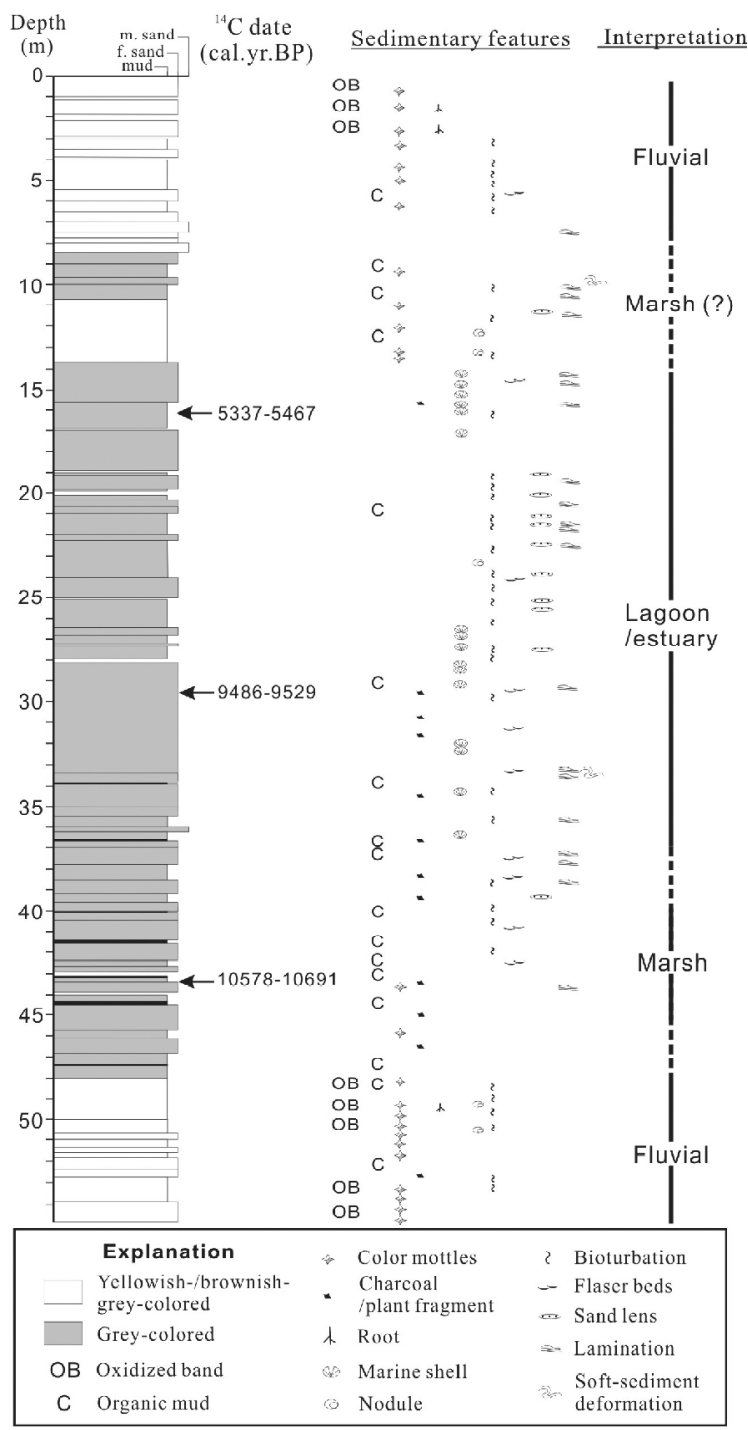

Fig. 3. Upper $55 \mathrm{~m}$ sequence of Lu-tsao core. For location see Fig. 1. Ground surface is $14.6 \mathrm{~m}$ above sea level. Sediments dated 9486 - 9529 cal.yr.BP ( $1 \sigma$ range) are interpreted as deposited -3 to $0 \mathrm{~m}$ of the coeval mean sea level, as they are rich in organic materials and contain marine shells. Sediments dated 10578-10691 cal.yr.BP are interpreted as deposited 0 to $3 \mathrm{~m}$ of the sea level, as they are associated with weakly-oxidized strata and are close in sequence to marine-shell-bearing strata. These two dates are used in testing eustatic sea level (Table 1). Date 5337-5467 cal.yr.BP is derived from marine-shell-bearing strata. However, as the sediments do not include abundant organic materials, their deposition position is not well constrained (merely $<0 \mathrm{~m}$ of the sea level). 
where subscripts $L$ and $U$, respectively, denote the lower and upper bounds of $U, A, D$, and $S_{e u}$ ( $S_{e u L}$ corresponds to $A_{U}$ during sea-level rise and to $A_{L}$ during sea-level fall).

Figure 4 plots the calculated "rock-uplift" rates with the dates that yield them. As the plot shows, the rates obtained are mostly above $-5 \mathrm{~mm} \mathrm{yr}^{-1}$, with exceptions occurring exclusively from the dates younger than $5 \mathrm{ka}$. Additionally, for the cores containing more than one date, the rates calculated by the dates younger than 5 ka were generally lower than those calculated by the other dates in the same cores (Fig. 4). This temporal change in the "uplift" rate was attributed to the compaction of the younger sediments, which was probably enhanced by excess ground-water withdrawal in the past few decades (i.e., the rates calculated by the dates $<5 \mathrm{ka}$ include the components of both true rock uplift and sediment compaction). These younger dates $(<5 \mathrm{ka})$ were therefore excluded in the following analysis.

\subsection{Testing for 11 - 5 ka Eustatic Sea Level}

The test focused on the cores containing more than one date (20 - $5 \mathrm{ka}$ ) derived from the coastal sediments (totally 28 cores with 67 dates) (Table 1 and Fig. 1). These were supplemented by data from one core and two outcrops (totally 7 dates) by Chen and Liu (2000) (Table 1 and Fig. 1). Applying Sea level SL-1 caused inconsistent rock-uplift rates for 14 sites (i.e., the calculated possible ranges of the rock-uplift rates in each of the 14 sites fail to overlap if Sea level SL-1 is applied). These apparently inconsistent rock-uplift rates did not show

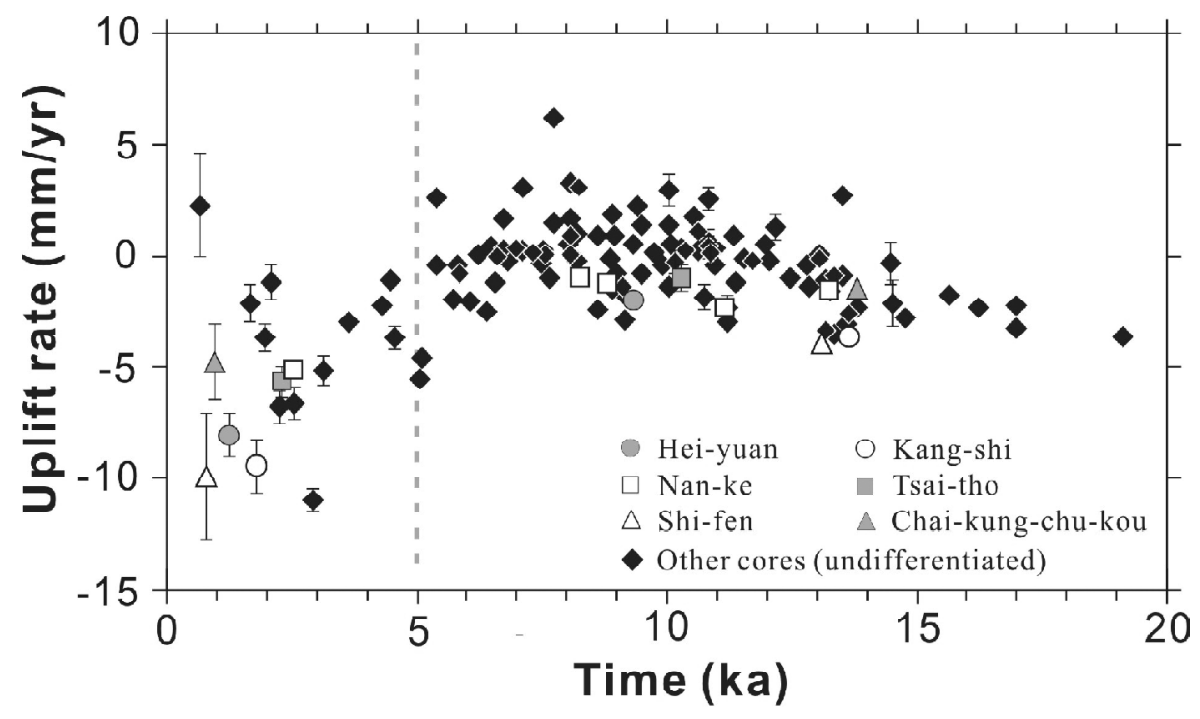

Fig. 4. Plot of uplift rates with the dates (median values) that yield them. The uplift rates are calculated by using Sea level SL-1 (Fig. 2) (possible ranges $>1 \mathrm{~mm} \mathrm{yr}^{-1}$ shown as bars). Data from the same cores (with at least one date $<5 \mathrm{ka}$ ) are specified. 
Table 1. Radiocarbon dates used in this study.

\begin{tabular}{|c|c|c|c|c|c|c|c|c|}
\hline \multicolumn{3}{|c|}{ Sites $^{I}$ (Fig. I for location) } & \multicolumn{3}{|c|}{ Sample } & \multirow{2}{*}{$\begin{array}{l}{ }^{14} \mathrm{C} \text { age } \\
(\text { yr. BP) }\end{array}$} & \multirow{2}{*}{$\begin{array}{c}\text { Calibrated age }^{3} \\
\text { (1б range) } \\
\text { (cal. yr. BP) }\end{array}$} & \multirow{2}{*}{$\begin{array}{c}\text { Range of rock } \\
\text { uplift rate } \\
(\mathrm{mm} / \mathrm{yr})\end{array}$} \\
\hline Name & $\begin{array}{c}\text { TWD67 } \\
\text { N (m) }\end{array}$ & $\begin{array}{c}\text { TWD67 } \\
\text { E (m) }\end{array}$ & $\begin{array}{c}\text { Elevation } \\
\text { (m) }\end{array}$ & Material & $\begin{array}{c}\text { Dep. } \\
\text { setting }\end{array}$ & & & \\
\hline \multirow{2}{*}{ Yuan-lin } & \multirow{2}{*}{2649675} & \multirow{2}{*}{206624} & -9.8 & wood & $\mathrm{L} / \mathrm{T}$ & $6670 \pm 70$ & $7475-7607$ & \multirow{2}{*}{$(-0.28,-0.08)$} \\
\hline & & & -11.6 & wood & $\mathrm{L} / \mathrm{T}$ & $6700 \pm 60$ & $7493-7654$ & \\
\hline \multirow{3}{*}{ Tian-yang } & \multirow{3}{*}{2624986} & \multirow{3}{*}{178688} & -5 & shell & $\mathrm{L} / \mathrm{T}$ & $5420 \pm 50$ & $5734-5856$ & \multirow{3}{*}{$(-0.92,-0.91)$} \\
\hline & & & -7.4 & shell & $L / T$ & $5470 \pm 50$ & $5781-5898$ & \\
\hline & & & -34.3 & wood & $\mathrm{M}$ & $9050 \pm 100$ & $9922-10394$ & \\
\hline \multirow{3}{*}{ Lun- $z u$} & \multirow{3}{*}{2611936} & \multirow{3}{*}{183344} & -8.9 & wood & $L / T$ & $7370 \pm 100$ & $8039-8325$ & \multirow{3}{*}{$(0.44,0.46)$} \\
\hline & & & -8.9 & shell & $L / T$ & $7620 \pm 80$ & $7987-8151$ & \\
\hline & & & 32.6 & peat & $\mathrm{M}$ & $9230 \pm 60$ & $10268 \cdot 10485$ & \\
\hline \multirow{2}{*}{ Sin-pi } & \multirow{2}{*}{2598383} & \multirow{2}{*}{181250} & -2.4 & wood & $\mathrm{M} / \mathrm{L} / \mathrm{T}$ & $6944 \pm 58$ & $7683-7820$ & \multirow{2}{*}{$(0.55,0.93)$} \\
\hline & & & -39.3 & wood & $\mathrm{M}$ & $9990 \pm 60$ & $11259-11442$ & \\
\hline \multirow{3}{*}{ Dong-shi } & & & -42.2 & shelt & $\mathrm{L} / \mathrm{T}$ & $8700 \pm 70$ & $9006-9412$ & \\
\hline & 2595712 & 162773 & -49.2 & organic mud & LT & $10200 \pm 80$ & $11642-12275$ & \\
\hline & & & -64.9 & organic mud & $\mathrm{M}$ & $10790 \pm 140$ & $12636-13006$ & \\
\hline Thu-wei & 2595590 & 171471 & -31.2 & organic mud & $\mathrm{M}$ & $9240 \pm 50$ & $10283-10302$ & $(-0,03 \quad 027)$ \\
\hline Doutwe & 20, & $1 / 1+11$ &. .50 .6 & organic mud & $\mathrm{M}$ & $9930+190$ & $11165-11950$ & $(-0.05,0.2)$ \\
\hline Nhn & 2500705 & 185076 & 13.5 & organic mud & $I / T$ & $4622 \pm 57$ & $5294-5465$ & $1002773)$ \\
\hline Tan-jung & 2590795 & $1859 / 6$ & -15.8 & wood & $\mathrm{M}$ & $9517 \pm 67$ & $10643-11067$ & $(2.02,2.75)$ \\
\hline Lu-tsao & 2590208 & 178269 & -15.1 & wood & $\mathrm{L} / \mathrm{T}$ & $8490 \pm 40$ & $9486-9529$ & $(0.99,1.15)$ \\
\hline Lu-tsao & 2590208 & 178269 & -28.8 & wood & $\mathrm{M}$ & $9420 \pm 40$ & $10578-10691$ & $(0.95,1.15)$ \\
\hline & 2581210 & 173556 & -40.6 & organic mud & $L / T$ & $9500 \pm 110$ & $10582-11071$ & \\
\hline An-ne1 & 2381210 & 173556 & -63.3 & organic mud & $\mathrm{M}$ & $11030 \pm 120$ & $12926-13152$ & $(-0.13,0.06)$ \\
\hline Chong-xi & 2576361 & 184263 & -4.3 & wood & $\mathrm{L} / \mathrm{T}$ & $7277 \pm 66$ & $7982-8164$ & $(142,149)$ \\
\hline Chong-xi & $25 / 6361$ & $1842 b 3$ & -21.7 & wood & $\mathrm{M} / \mathrm{L} / \mathrm{T}$ & $9345 \pm 58$ & $10429-10669$ & $(1.42,1.49)$ \\
\hline & & & -13 & wood & $\mathrm{L} / \mathrm{T}$ & $7201 \pm 66$ & $7883-8146$ & \\
\hline Liots-ying & 2574934 & 178839 & -29.6 & shell & $L / T$ & $9129 \pm 58$ & 9574-9925 & $(-0.24,0.16)$ \\
\hline & 2570038 & 173545 & -64.2 & wood & $\mathrm{L} / \mathrm{T}$ & $9914: 61$ & $11204-11543$ & \\
\hline Xia-ying & $25 / 0238$ & 175545 & -79.2 & peat & $\mathrm{L} / \mathrm{T}$ & $10873-60$ & $12679-13013$ & $(-1.44,-1.09)$ \\
\hline & & & -37.9 & wood & $L / T$ & $8170 \pm 160$ & $8812-9423$ & \\
\hline & & 174375 & -51.7 & shell & $\mathrm{L} / \mathrm{T}$ & $8627 \pm 59(?)$ & $8962-9376$ & \\
\hline Zong-ye & 2565599 & $1742 / 5$ & -71.3 & organic mud & $\mathrm{L} / \mathrm{T}$ & $10440 \pm 110$ & $12118-12790$ & $(-1.25,-1.16)$ \\
\hline & & & -89.0 & organic mud & $\mathrm{M}$ & $12210 \perp 160$ & $13847-15077$ & \\
\hline Fia-bra & 2560030 & 170055 & -4.7 & wood & $\mathrm{M}$ & $8050 \pm 40$ & $8784-9027$ & \\
\hline Ja-ba & 2500020 & 179950 & -31.5 & organic mud & $\mathrm{M}$ & $11510 \pm 50$ & $13206-13792$ & \\
\hline Shum & 2550701 & 176200 & -9.4 & wood & $\mathrm{L} / \mathrm{T}$ & $6204 \pm 63$ & $7010-7208$ & \\
\hline Shan-łua & 2559721 & 176200 & -35.5 & wood & $L / T$ & $8544=64$ & $9474-9551$ & $(-0.80,-0.78)$ \\
\hline & & & -16.4 & shell & $\mathrm{L} / \mathrm{T}$ & $6956 \pm 77$ & $7404-7539$ & \\
\hline Xi-gang & 2558381 & 167156 & -116.3 & organic mud & $\mathrm{M}$ & $12270 \pm 270$ & $13851-15170$ & $(-1.25,-1.07)$ \\
\hline & & & -27.8 & shell & $\mathrm{L} / \mathrm{T}$ & $7850 \pm 60$ & $8239-8368$ & \\
\hline Nan-kr & 2556230 & 175430 & -35.0 & shell & $\mathrm{L} / \mathrm{T}$ & $8330 \pm 50$ & $8773-8916$ & \\
\hline Nan-ke & 2570230 & $1 / 2430$ & -74.8 & wood & $\mathrm{L} / \mathrm{T}$ & $9770 \pm 70(?)$ & $11119-11232$ & $(-1.00,-1.37)$ \\
\hline & & & -88.6 & wood & $L / T$ & $11270 \pm 40$ & $13148-13386$ & \\
\hline Wan-xinor & 2553700 & & -114.5 & organic mud & $L / T$ & $11560 \pm 110$ & $13405-13808$ & \\
\hline Nan-xing & 2533702 & 169053 & -144.8 & organic mud & $\mathrm{L} / \mathrm{T}$ & $14060=130$ & $16580-17434$ & \\
\hline
\end{tabular}




\begin{tabular}{|c|c|c|c|c|c|c|c|c|}
\hline \multirow{2}{*}{ Xing-shi } & \multirow{2}{*}{2553431} & \multirow{2}{*}{177117} & -11.7 & wood & M & $6400 \pm 40$ & $7270-7414$ & \multirow{2}{*}{$(-1.19,-0.66)$} \\
\hline & & & $\ldots-31.7$ & wood & $\mathrm{L} / \mathrm{T}$ & $8090 \pm 130$ & $8772-\cdots 9256$ & \\
\hline \multirow{3}{*}{ Fan-zu-liao } & \multirow{3}{*}{2550542} & \multirow{3}{*}{178388} & -16.3 & wood & $\mathrm{L} / \mathrm{T}$ & $8080 \pm 40$ & $8818-9125$ & \multirow{3}{*}{$(0.30,0.30)$} \\
\hline & & & -27.9 & wood & $\mathrm{L} / \mathrm{T}$ & $8750 \pm 50$ & $9603-9889$ & \\
\hline & & & -44.5 & wood & M & $9710 \pm 60$ & $10890-11198$ & \\
\hline \multirow{2}{*}{ An-qiing } & \multirow{2}{*}{2549095} & \multirow{2}{*}{166368} & -130.8 & organic mud & $\mathrm{L} / \mathrm{T}$ & $13010 \pm 40$ & $15339-15960$ & \\
\hline & & & -163.6 & organic nud & $\mathrm{L} / \mathrm{T}$ & $14160 \pm 50$ & $16792-17218$ & \\
\hline \multirow{4}{*}{ Chang-xing } & \multirow{4}{*}{2543532} & \multirow{4}{*}{173232} & -22.9 & shell & $\mathrm{L} / \mathrm{T}$ & $7220 \pm 60$ & $7615-7727$ & \multirow{4}{*}{$(-1.46,-1.45)$} \\
\hline & & & -36.7 & wood & $\mathrm{L} / \mathrm{T}$ & $8030 \pm 60$ & $8776-9025$ & \\
\hline & & & -63.0 & shell & $\mathrm{L} / \mathrm{T}$ & $9960 \pm 60$ & $10387-11081$ & \\
\hline & & & -81.0 & shell & $\mathrm{L} / \mathrm{T}$ & $11680 \pm 40$ & $13006-13345$ & \\
\hline \multirow{3}{*}{$\begin{array}{l}\text { *Tai-nan } \\
\text { terrace }\end{array}$} & \multirow{3}{*}{2544675} & \multirow{3}{*}{171056} & 22.60 & wood & $\mathrm{L} / \mathrm{T}$ & $5840 \leq 50$ & $6566-6725$ & \multirow{3}{*}{$(3.52,3.77)$} \\
\hline & & & 20.30 & wood & $\mathrm{L} / \mathrm{T}$ & $5930 \pm 50$ & $6671-6847$ & \\
\hline & & & 17.70 & wood & $\mathrm{L} / \mathrm{T}$ & $6950+150$ & $7662-7936$ & \\
\hline \multirow{3}{*}{ Ren-de } & \multirow{3}{*}{2539533} & \multirow{3}{*}{173284} & -25.1 & shell & $\mathrm{L} / \mathrm{T}$ & $8350 \pm 60$ & $8786-8935$ & \multirow{3}{*}{$(-0.73,-0.62)$} \\
\hline & & & -36.8 & shell & $\mathrm{L} / \mathrm{T}$ & $9170 \pm 60$ & $9600-10270$ & \\
\hline & & & -81.4 & shell & $\mathrm{L} / \mathrm{T}$ & $11940 \pm 70$ & $13178-13489$ & \\
\hline \multirow{2}{*}{$\begin{array}{l}\text { *1)awan } \\
\text { low land }\end{array}$} & \multirow{2}{*}{2538044} & \multirow{2}{*}{171184} & -10.20 & shell & $\mathrm{L} / \mathrm{T}$ & $5300+50$ & $5600-5696$ & \multirow{2}{*}{$(-2.08,-1.87)$} \\
\hline & & & -45.90 & shell & $\mathrm{L} / \mathrm{T}$ & $8850 \pm 80$ & $9086-9552$ & \\
\hline \multirow{2}{*}{$\begin{array}{c}\text { *Na-yi } \\
\text { golf course }\end{array}$} & \multirow{2}{*}{2535466} & 180750 & 27.5 & shell & $\mathrm{L} / \mathrm{T}$ & $5840 \pm 50$ & $6199-6289$ & $(435443)$ \\
\hline & & & 15.5 & wood & $\mathrm{L} / \mathrm{T}$ & $8110 \pm 50$ & $9004-9220$ & $(4.35,4.43)$ \\
\hline San-min & 2504460 & 176306 & -3.1 & wood & $\mathrm{M}$ & $5920 \pm 40$ & $6671-6788$ & $(-0>20-016)$ \\
\hline $\operatorname{san}-\min$ & 2004400 & 170300 & -64.1 & sheil & $\mathrm{L} / \mathrm{T}$ & $11520 \pm 40$ & $12934-13145$ & $(-0.20,-0.16)$ \\
\hline & & & -4.5 & shell & $\mathrm{L} / \mathrm{T}$ & $5800 \pm 60$ & $6166-6276$ & $(-058-031)$ \\
\hline Wu-quan & 2502736 & 179610 & -59.1 & wood & $\mathrm{L} / \mathrm{T}$ & $10250 \pm 60$ & $11753-12307$ & $(-0.58,-0.31)$ \\
\hline Ming-guan & 2501310 & 178355 & -12.0 & wood & $\mathrm{M}$ & $5750 \pm 40$ & $6491--6623$ & $(-232-178)$ \\
\hline Ming-quan & 2501310 & 178353 & -74.8 & sheil & $\mathrm{I} / \mathrm{T}$ & $10230+160$ & $10825-11604$ & $(-2.32,-1.78)$ \\
\hline Zhong-zhou & 2497871 & 176589 & -16.4 & wood & $\mathrm{L} / \mathrm{T}$ & $5300=55$ & $5991-6171$ & $(-25)-227)$ \\
\hline & & & -143.1 & sholl & $\mathrm{L} / \mathrm{T}$ & $14020 \pm 60$ & $16004-16471$ & $(-2.51,-2.27)$ \\
\hline Fo-gong & & 179444 & -8.3 & sheil & $\mathrm{L} / \mathrm{T}$ & $6190+110$ & $6487-6739$ & $(-130-0.55)$ \\
\hline Fo-gong & 2497275 & 179444 & 82.9 & sheil & $\mathrm{L} / \mathrm{T}$ & $12000 \pm 40$ & $13199 \cdots 13796$ & $(-1.30,-0.55)$ \\
\hline
\end{tabular}

$1 *$ Tai-nan terrace, *Da-wan low land, and *Nan-yi golf course from Chen and Liu (2000), others from the Central Geological Survey.

${ }^{2} \mathrm{~L} / \mathrm{T}$ : lagoon or tidal flat; M: coastal marsh.

${ }^{3}$ using program of CALIB REV4.4.2 (Stuiver and Reimer 1993).

${ }^{4}$ calculated by using sea level shown in Fig. 6. Blank where calculated rates from different dates do not overlap. 
systematic patterns. At many sites, the rates calculated from the younger dates were greater than those calculated from the older dates, which cannot be due to sediment compaction. The rock uplift of the study sites was assumed to have been steady. The analytical results therefore show that either the sea level was not SL-1, or the dates derived from the 14 sites were erroneous.

The 11 - 5 ka part of SL-1 (and fixing other parts) was adjusted to determine whether it would fit more of the data than the original SL-1. To simplify the mathematical expression, this part of the sea level ( $2.3 \mathrm{~m}$ at $5 \mathrm{ka}$ and $-46.5 \mathrm{~m}$ at $11 \mathrm{ka})$ was assumed to comprise three straight segments with nodes at time $x_{0}$ and $x_{1}$ (in ka) (Fig. 5), which can be formulated as:

$$
\begin{array}{ll}
S_{\mathrm{eu}}=-(\mathrm{x}-5) a+2.3 & \left(5<\mathrm{x}<x_{0}\right), \\
S_{\mathrm{eu}}=-\left(\mathrm{x}-x_{0}\right) b+y_{0} & \left(x_{0}<\mathrm{x}<x_{1}\right), \\
S_{\mathrm{eu}}=-(\mathrm{x}-11) c-46.5 & \left(x_{1}<\mathrm{x}<11\right),
\end{array}
$$

where $\mathrm{x}$ denotes time (in ka), $y_{0}$ denotes the sea-level position (in m) at $x_{0} ; a, b$, and $c$, respectively, are gradients (absolute values, in $\mathrm{m} \mathrm{ky}^{-1}$ ) of the sea-level curves in the given time periods (Fig. 5). Linking these three segments at $x_{0}$ and $x_{1}$ yields:

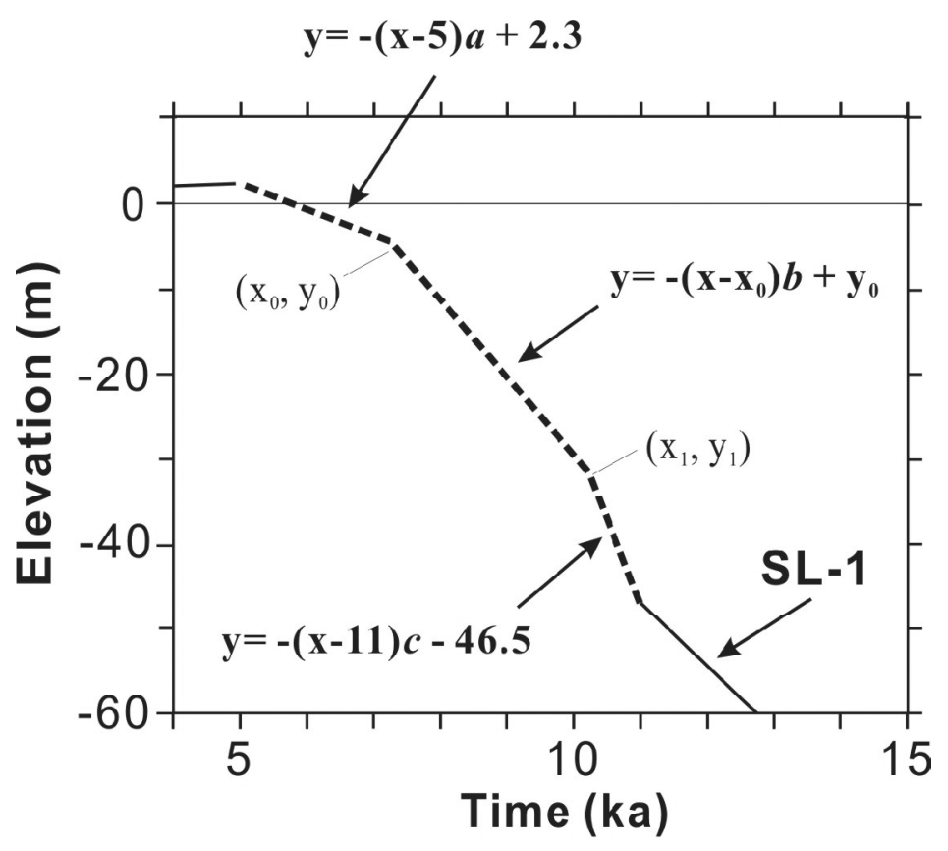

Fig. 5. Parameters of sea-level curve tested in this study. 


$$
\begin{aligned}
& a=\left(y_{0}-2.3\right) /\left(x_{0}-5\right), \\
& c=\frac{\left(x_{1}-x_{0}\right) b+y_{0}-46.5}{\left(x_{1}-11\right)} .
\end{aligned}
$$

According to Eqs. 4 and 5, the lower and upper bounds of the rock uplift rate can be rewritten as:

$$
\begin{array}{ll}
U_{L}=\frac{E+\left(A_{L}-5\right) a-2.3-D_{U}}{A_{L}} & \left(5<A_{L}<x_{0}\right), \\
U_{L}=\frac{E+\left(A_{L}-x_{0}\right) b-y_{0}-D_{U}}{A_{L}} & \left(x_{0}<A_{L}<x_{1}\right), \\
U_{L}=\frac{E+\left(A_{L}-11\right) c+46.5-D_{U}}{A_{L}} & \left(x_{1}<A_{L}<11\right),
\end{array}
$$

and

$$
\begin{array}{ll}
U_{U}=\frac{E+\left(A_{U}-5\right) a-2.3-D_{L}}{A_{U}} & \left(5<A_{L}<x_{0}\right), \\
U_{U}=\frac{E+\left(A_{U}-x_{0}\right) b-y_{0}-D_{L}}{A_{U}} & \left(x_{0}<A_{U}<x_{1}\right), \\
U_{U}=\frac{E+\left(A_{U}-11\right) c+46.5-D_{L}}{A_{U}} & \left(x_{1}<A_{U}<11\right) .
\end{array}
$$

The steady rock uplift requires that the uncertainty ranges of the rock uplift rates (bounded by $U_{L}$ and $U_{U}$ ) calculated at the same core overlap, or:

$$
U_{L 1} \leq U_{U 2} \text { and } U_{U 1} \geq U_{L 2},
$$

where subscripts 1 and 2 denote any parameters $(U, A, D$, and $E)$ at arbitrary 1 and 2 stratigraphic positions in a core.

Equations 6 to 9 provide bases to solve the ranges of $a, b$, and $c$ for given $x_{0}, x_{1}$, and $y_{0}$. For example, where all $A_{L 1}, A_{U 1}, A_{L 2}, A_{U 2}$ are within the time period of $x_{0}$ and $x_{1}(=10 \mathrm{ka})$, 
and $y_{0}=0$, we obtain:

$$
\frac{\left(E_{2}-D_{L 2}\right) / A_{U 2}-\left(E_{1}-D_{U 1}\right) / A_{L 1}}{\left(A_{U 2}-x_{0}\right) / A_{U 2}-\left(A_{L 1}-x_{0}\right) / A_{L 1}} \leq b \leq \frac{\left(E_{2}-D_{U 2}\right) / A_{L 2}-\left(E_{1}-D_{L 1}\right) / A_{U 1}}{\left(A_{L 2}-x_{0}\right) / A_{L 2}-\left(A_{U 1}-x_{0}\right) / A_{U 1}}
$$

or

$$
\begin{aligned}
\frac{\left(E_{2}+46.5 A_{U 2}-465-D_{L 2}\right) / A_{U 2}-\left(E_{1}-D_{U 1}\right) / A_{L 1}}{} \leq b \\
\left(A_{0}-10\right)\left(A_{U 2}-11\right) / A_{U 2} \\
\leq \frac{\left(E_{2}+46.5 A_{L 2}-465-D_{U 2}\right) / A_{L 2}-\left(E_{1}-D_{L 1}\right) / A_{U 1}}{\left(A_{0}-10\right)\left(A_{L 2}-11\right) / A_{L 2}-\left(A_{U 1}-A_{0}\right) / A_{U 1}},
\end{aligned}
$$

where $x_{0}<A_{L 1}<A_{U 1}<10 \mathrm{ka}, 10 \mathrm{ka}<A_{L 2}<A_{U 2}<11 \mathrm{ka}$, and $y_{0}=0$.

Similarly, when $x_{0}<A_{L 1}<A_{U 1}<10 \mathrm{ka}, 11 \mathrm{ka}<A_{L 2}<A_{U 2}$, and $y_{0}=0$, the range of $b$ has the general form of:

$$
\frac{U_{L 2} A_{U 1}-E_{1}+D_{L 1}}{x_{0}-A_{U 1}} \leq b \leq \frac{U_{U 2} A_{L 1}-E_{1}+D_{U 1}}{x_{0}-A_{L 1}}
$$

Here, $U_{L 2}$ and $U_{U 2}$ are the lower and upper bounds of the uplift rates calculated by applying the sea level derived from the Sunda Shelf. Notably, these calculations may reverse the comparative relationship in the equations, depending on the sign of the denominators (i.e., in some cases only maximal or minimal values of $a, b$, and $c$ can be obtained). The values of $x_{0}, x_{1}$, and $y_{0}$ were progressively changed, with an interval of $0.01 \mathrm{ka}$, to calculate the ranges of $a, b$, and $c$ for each core. The overlap of the ranges of these variables obtained from different cores was then determined.

\section{RESULTS}

Tables 2 and 3 summarize the experimental results. Parameter $y_{0}=0$ was set, and $x_{0}, x_{1}$, $b$, and $c$ were used to describe the sea-level curves (the results of changing $y_{0}$ with fixed $x_{0}$ were similar to those of changing $x_{0}$ with $y_{0}=0$, and are not presented herein). As shown, the applicability of the sea-level curves depends on the value of $x_{0}$ (and therefore $b$ ). The minimum number of the dates that would yield inconsistent uplift rates falls as $x_{0}$ rises, and reaches a minimum around $6.5 \mathrm{ka} \leq x_{0} \leq 6.6 \mathrm{ka}$. As $x_{0}$ rises, $b$ also rises, from $7.0 \mathrm{~m} \mathrm{ky}^{-1}$ for SL-1 to $8.2-9.4 \mathrm{~m} \mathrm{ky}^{-1}$ when $6.5 \mathrm{ka} \leq x_{0} \leq 6.6 \mathrm{ka}$ (Table 3 ). The optimal sea-level curves are obtained when $6.53 \mathrm{ka} \leq x_{0} \leq 6.59 \mathrm{ka}, 9.91 \mathrm{ka} \leq x_{1} \leq 10.02 \mathrm{ka}$, and $8.29 \mathrm{~m} \mathrm{ky}^{-1} \leq b \leq 8.46 \mathrm{~m} \mathrm{ky}^{-1}$, which reduces the minimum number of the apparently erroneous dates to seven (i.e., all but seven dates result in consistent uplift rates if the sea-level data are applied). Figure 6 illustrates 
Table 2. Minimum number of dates failing to fit into sea-level curves tested.

\begin{tabular}{|c|c|c|c|c|c|c|c|c|}
\hline$x_{I}$ & $6.0 \mathrm{ka}$ & $6.1 \mathrm{ka}$ & $6.2 \mathrm{ka}$ & $6.3 \mathrm{ka}$ & $6.4 \mathrm{ka}$ & $6.5 \mathrm{ka}$ & $6.6 \mathrm{ka}$ & $6.7 \mathrm{ka}$ \\
\hline $9.0 \mathrm{ka}$ & 13 & 12 & 11 & 10 & 10 & 10 & 11 & 11 \\
\hline $9.2 \mathrm{ka}$ & 11 & 11 & 11 & 10 & 10 & 9 & 10 & 10 \\
\hline $9.4 \mathrm{ka}$ & 12 & 11 & 10 & 10 & 10 & 9 & 10 & 10 \\
\hline $9.6 \mathrm{ka}$ & 12 & 12 & 11 & 9 & 9 & 9 & 10 & 10 \\
\hline $9.8 \mathrm{ka}$ & 12 & 12 & 11 & 10 & 9 & 8 & 8 & 10 \\
\hline $10.0 \mathrm{ka}$ & 12 & 12 & 11 & 10 & 10 & 9 & 8 & 10 \\
\hline $10.2 \mathrm{ka}$ & 12 & 12 & 11 & 10 & 10 & 9 & 9 & 9 \\
\hline $10.4 \mathrm{ka}$ & 13 & 13 & 12 & 11 & 11 & 10 & 10 & 10 \\
\hline $10.6 \mathrm{ka}$ & 13 & 13 & 12 & 11 & 11 & 10 & 10 & 11 \\
\hline $10.8 \mathrm{ka}$ & 13 & 13 & 12 & 11 & 11 & 10 & 10 & 11 \\
\hline
\end{tabular}

one optimal sea-level curve ( $x_{0}=6.55 \mathrm{ka}, x_{1}=10.00 \mathrm{ka}, b=8.35 \mathrm{~m} \mathrm{ky}^{-1}$, and $\left.c=17.70 \mathrm{~m} \mathrm{ky}^{-1}\right)$ and the data constraining it (Table 1 shows the corresponding rock uplift rates).

In this test, the assigned $b$ values were always smaller than $c$ values to achieve a meaningful data fit (Table 3), indicating that during a period since $\sim 11 \mathrm{ka}$, the eustatic sea level rose faster than the rest of the post-glacial era. The duration and magnitude of this rapid sea-level rise, however, are not well constrained, as the value of $x_{1}$ (and its corresponding $c$ ) affects the result of this test less than the value of $x_{0}$ (e.g., changing $x_{1}$ by $1 \mathrm{ky}$ and doubling $c$ values only changes the minimum number of apparently wrong dates by 1 or 2) (Tables 2,3 ).

\section{DISCUSSION}

This study found de-glacial sea-level curves that fit the majority of the data reported from the Western Coastal Plain, under the assumption of steady rock uplift. The minimum seven dates (from six sites) that failed to be accommodated by the curves were examined in detail (Table 1). Two of these dates came from the Nanxing and Anqing cores, each of which contained two dates older than $11 \mathrm{ka}$; two were among the three dates (two of which were older than $11 \mathrm{ka}$ ) at the Dongshi core, and the other three were obtained from the Nanke, Zongye, and Jiaba cores. Among these seven dates, only the date from Jiaba could pass the test by adjusting the sea-level curve parameters, which, however, would cause additional problems for at least two dates from other cores. No sea-level curves tested in this study could fit the other six dates, even when the $2 \sigma$ ranges of the calibrated ages were applied. 
Table 3. Optimal gradients ( $b$ and $c$ ) of sea-level curves for given $x_{0}$ and $x_{1}$.

\begin{tabular}{|c|c|c|c|c|c|}
\hline$X_{X_{I}} X^{X_{0}}$ & $6.3 \mathrm{ka}$ & $6.4 \mathrm{ka}$ & $6.5 \mathrm{ka}$ & $6.6 \mathrm{ka}$ & $6.7 \mathrm{ka}$ \\
\hline $9.0 \mathrm{ka}$ & $\begin{array}{l}b=7.57-7.68 \\
c=12.88-13.03\end{array}$ & $\begin{array}{l}b=7.83-8.14 \\
c=12.67-13.07\end{array}$ & $\begin{array}{l}b=8.14-8.46 \\
c=12.68-13.08\end{array}$ & $\begin{array}{l}b=8.47-8.81 \\
c=12.68-13.09\end{array}$ & $\begin{array}{l}b=8.849 .20 \\
c=12.67-13.08\end{array}$ \\
\hline $9.2 \mathrm{ka}$ & $\begin{array}{l}b=7.57-7.68 \\
c=13.46-13.64\end{array}$ & $\begin{array}{l}b=7.83-8.05 \\
c=13.31-13.65\end{array}$ & $\begin{array}{l}b=8.14-8.35 \\
c=13.31-13.62\end{array}$ & $\begin{array}{l}b=8.47-8.67 \\
c=13.31-13.60\end{array}$ & $\begin{array}{l}b=8.84-9.02 \\
c=13.31-13.56\end{array}$ \\
\hline $9.4 \mathrm{ka}$ & $\begin{array}{l}b=7.57-7.68 \\
c=14.18-14.40\end{array}$ & $\begin{array}{c}b=7.83-8.22 \\
\text { or } 8.39-8.40 \\
c=13.65-14.38 \\
\text { or } 13.13-13.33\end{array}$ & $\begin{array}{l}b=8.14-8.58 \\
c=13.51-14.31\end{array}$ & $\begin{array}{l}b=9.00-9.00 \\
c=13.31-13.31\end{array}$ & $\begin{array}{c}b=8.50-8.71 \\
\quad \text { or } 8.84-9.34 \\
c=14.36-14.72 \\
\text { or } 13.30-14.15\end{array}$ \\
\hline $9.6 \mathrm{ka}$ & $\begin{array}{l}b=7.57-7.68 \\
c=15.11-15.37\end{array}$ & $\begin{array}{l}b=7.96-8.22 \\
c=14.43-14.87\end{array}$ & $\begin{array}{l}b=8.77-8.99 \\
c=13.31-13.80\end{array}$ & $\begin{array}{c}b=8.47-8.49 \\
\quad \text { or } 9.00-9.29 \\
c=15.02-15.06 \\
\text { or } 13.31-13.93\end{array}$ & $\begin{array}{c}b=8.50-8.71 \\
\quad \text { or } 8.84 .9 .41 \\
c=15.17-15.61 \\
\text { or } 13.72-14.90\end{array}$ \\
\hline $9.8 \mathrm{ka}$ & $\begin{array}{l}b=7.57-7.68 \\
c=16.35-16.67\end{array}$ & $\begin{array}{l}b=7.83-8.10 \\
c=15.80-16.57\end{array}$ & $\begin{array}{l}b=8.38-8.58 \\
c=15.16-15.71\end{array}$ & $\begin{array}{l}b=9.24-9.37 \\
c=13.76-14.11\end{array}$ & $\begin{array}{c}b=8.50-8.71 \\
\quad \text { or } 8.84-9.41 \\
c=16.25-16.79 \\
\text { or } 14.44-15.91\end{array}$ \\
\hline $10.0 \mathrm{ka}$ & $\begin{array}{l}b=7.57-7.68 \\
c=18.08-18.49\end{array}$ & $\begin{array}{l}b=7.83-8.10 \\
c=17.34-18.31\end{array}$ & $\begin{array}{l}b=8.38-8.58 \\
c=16.47-17.17\end{array}$ & $\begin{array}{c}b=8.47-8.49 \\
\quad \text { or } 9.00-9.24 \\
c=17.63-17.70 \\
\text { or } 15.08-15.9\end{array}$ & $\begin{array}{c}b=8.50-8.71 \\
\quad \text { or } 8.84-9.41 \\
c=17.76-18.45 \\
\text { or } 15.45-17.33\end{array}$ \\
\hline $10.2 \mathrm{ka}$ & $\begin{array}{l}b=7.57-7.68 \\
c=20.68-21.22\end{array}$ & $\begin{array}{l}b=7.83-8.10 \\
c=19.65-20.93\end{array}$ & $\begin{array}{l}b=8.38-8.58 \\
c=18.44-19.37\end{array}$ & $\begin{array}{c}b=8.47-8.49 \\
\text { or } 9.00-9.00 \\
c=19.92-20.01 \\
\text { or } 17.61-17.62\end{array}$ & $\begin{array}{c}b=8.50-8.71 \\
\quad \text { or } 8.84-9.41 \\
c=20.02-20.94 \\
\text { or } 16.96-19.45\end{array}$ \\
\hline $10.4 \mathrm{ka}$ & $\begin{array}{l}b=7.57-7.68 \\
c=25.02-25.77\end{array}$ & $\begin{array}{l}b=7.83-8.10 \\
c=23.50-25.30\end{array}$ & $\begin{array}{l}b=8.38-8.58 \\
c=21.73-23.03\end{array}$ & $\begin{array}{c}b=8.26-8.32 \\
\text { or } 8.47-8.49 \\
c=24.81-25.19 \\
\text { or } 23.73-23.86\end{array}$ & $\begin{array}{c}b=8.50-8.71 \\
\text { or } 8.84-9.41 \\
c=23.79-25.08 \\
\text { or } 19.47-22.99\end{array}$ \\
\hline $10.6 \mathrm{ka}$ & $\begin{array}{l}b=7.57-7.68 \\
c=33.69-34.87\end{array}$ & $\begin{array}{l}b=7.83-8.10 \\
c=31.20-34.04\end{array}$ & $\begin{array}{l}b=8.38-8.58 \\
c=28.31-30.36\end{array}$ & $\begin{array}{l}b=8.47-8.49 \\
c=31.35-31.55\end{array}$ & $\begin{array}{c}b=8.50-8.71 \\
\quad \text { or } 8.84-9.41 \\
c=31.33-33.38 \\
\text { or } 24.50-30.06\end{array}$ \\
\hline $10.8 \mathrm{ka}$ & $\begin{array}{l}b=7.57-7.68 \\
c=59.70 \quad 62.18\end{array}$ & $\begin{array}{l}b=7.83-8.10 \\
c=54.30 \quad 60.24\end{array}$ & $\begin{array}{l}b=8.38-8.58 \\
c=48.03-52.33\end{array}$ & $\begin{array}{l}b=8.47-8.49 \\
c=54.21-54.63\end{array}$ & $\begin{array}{l}b=8.50-8.71 \\
c=53.95-58.25\end{array}$ \\
\hline
\end{tabular}

The eustatic sea-level curve derived from the Sunda Shelf has been assumed to be applicable in Taiwan, implying that either or both of the dates older than $11 \mathrm{ka}$ from each of the Nanxing, Anqing and Dongshi cores are incorrect. Fitting the apparently erroneous dates at Zongye (8962-9376 cal. yr.B.P.) and Nanke (1119-11232 cal. yr.B.P.) requires eustatic sealevel curves more complicated than those used in the test. However, over the periods defined by the two dates, the obtained optimal sea-level curves were well constrained by multiple dates from other cores (Fig. 6) (i.e., making sea-level curves applicable for Zongye and Nanke will cause problems for many other cores). Both the Zongye and Nanke cores show unsteady deposition rates, if the erroneous dates are included (Fig. 7). Excluding these dates then makes 


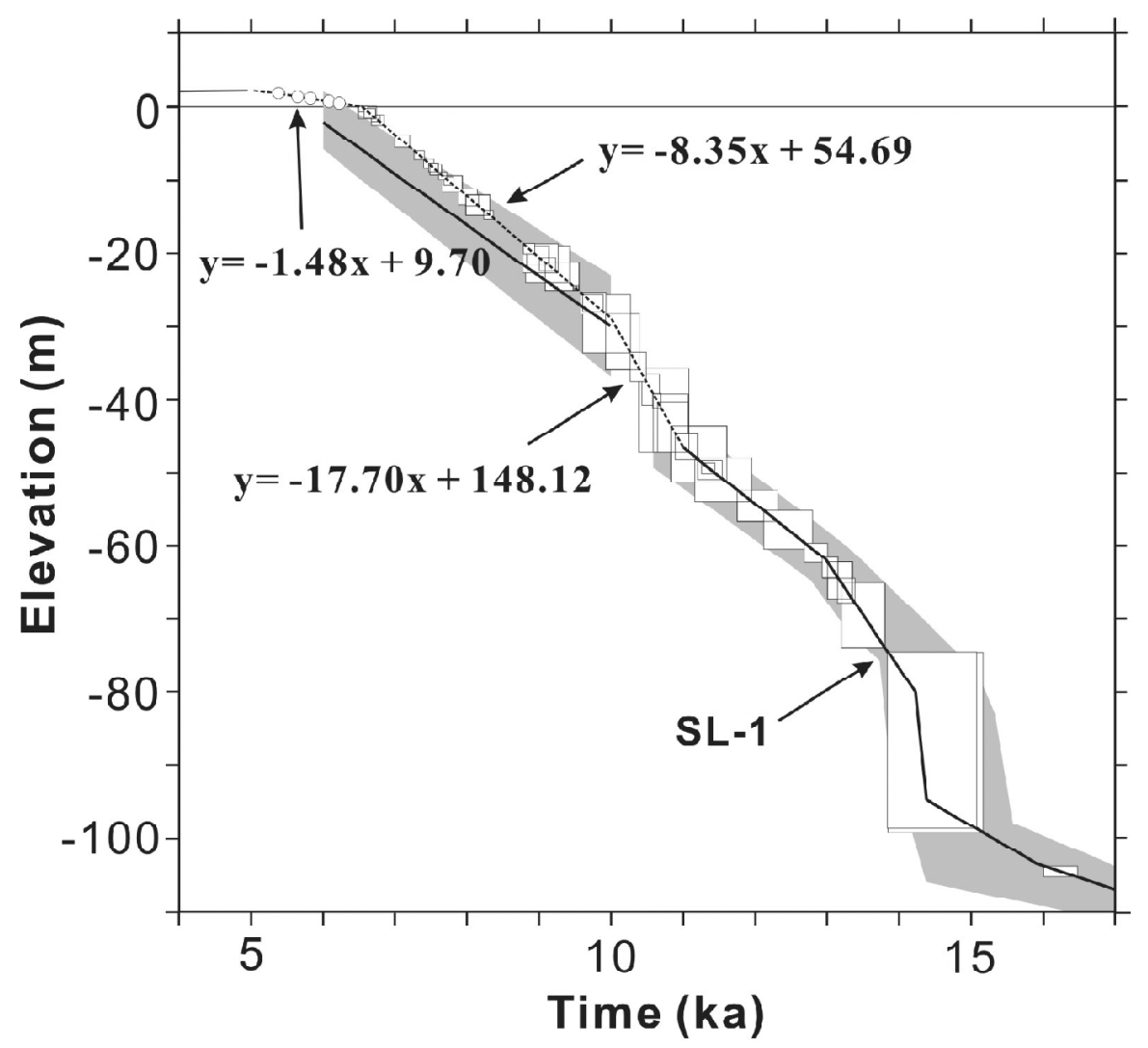

Fig. 6. One optimal sea-level curve obtained in this study (dashed). Boxes show $1 \sigma$ ranges of dates $(>6.5 \mathrm{ka})$ and their corresponding sea-level ranges that result in consistent rock-uplift rates (see Table 1 for values). Circles are median values of both dates $(<6.5 \mathrm{ka})$ and sea-level ranges. Solid lines and shaded areas as in Fig. 2.

the deposition rates of both cores nearly constant during $\sim 14$ to $8 \mathrm{ka}$ (Fig. 7).

The data set includes only 5 sites with more than one date (totally 10 dates) older than 11 ka (Table 1). Among these dates, the data from the Nanxing, Anqing and Dongshi sites failed to fit into the sea-level curve derived from the Sunda Shelf, emphasizing that the applicability of the Sunda Shelf's sea level to Taiwan, although assumed applicable, has not been strictly tested. Still, 17 sites (more than half of the study sites) contain at least two dates (totally 39 dates) within 11 - $5 \mathrm{ka}$ (Table 1). The eustatic sea level constructed by these dates is independent of the data from the Sunda Shelf (except that the sea level was assumed to be $-46.5 \mathrm{~m}$ at $11 \mathrm{ka}$ ). These data indicate a relatively rapid sea-level rise occurring around the onset of the post-glacial period, which is consistent with the sea-level data observed at Barbados (the meltwater pulse 1B; Fairbanks 1989). 


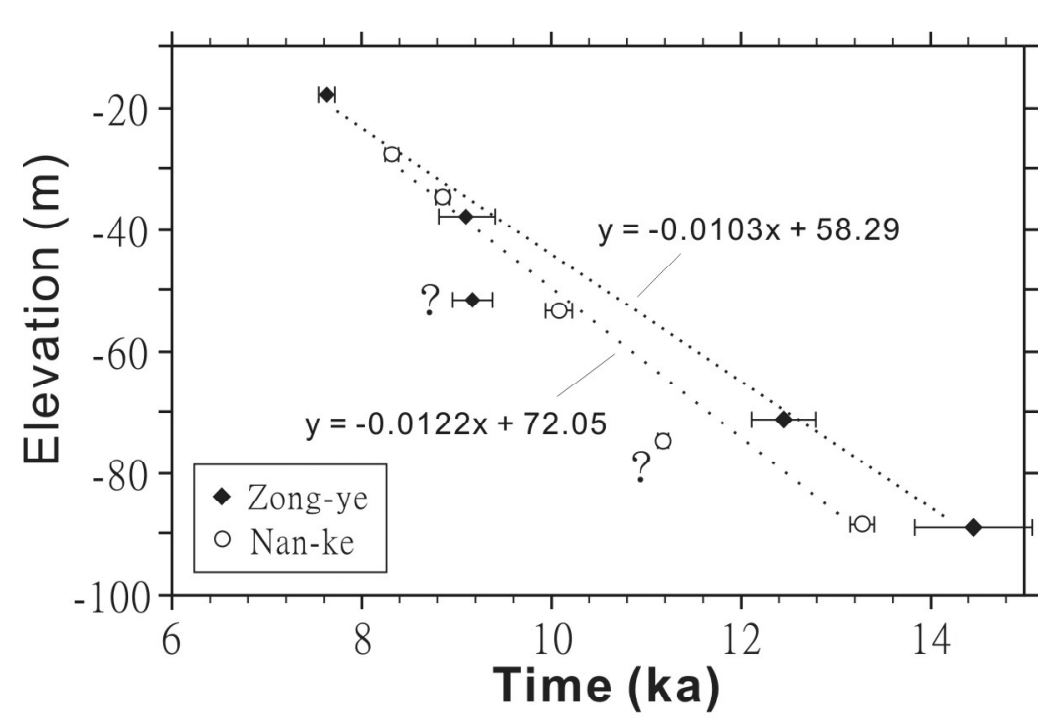

Fig. 7. Plots of dates with their elevations for Zongye and Nanke cores. Bars show $1 \sigma$ ranges of the dates. Question marks point out dates that are apparently incorrect.

The optimal sea-level curves obtained in this study show that the rise of the post-glacial sea level should have significantly slowed down (or formed a plateau) before reaching the climax at ca. $5 \mathrm{ka}$. The optimal time of this sea-level deflection is about $6.5-6.6 \mathrm{ka}$. On the actively uplifting eastern coast of Taiwan, beach sediments at the inner edges of the major marine terraces have been dated 5.7 - 6.4 ka at Pa-hsien-tung (Sung 1969) and $6.4-6.6 \mathrm{ka}$ at Hsiao-ma (Huang 1991). As these dates are derived from archeological relics, the deposition of these beach sediments is likely to have preceded these dates. Under the rapid rock uplift, the timing of deposition of these sediments, which records the positions of the Holocene relative sea-level high stand, is sensitive to the deflection of the post-glacial sea-level rise. The dates at Pahsientung and Hsiaoma indicate that this deflection happened no later than $6.4-6.6 \mathrm{ka}$, which is consistent with the analytical results of this study.

Hsieh et al. (2004) used Sea level SL-1 and its assumed uncertainties to calculate rockuplift rates on the eastern coast of Taiwan, which are commonly $6-8 \mathrm{~m} \mathrm{ky}^{-1}$. These rates are close to the $7 \mathrm{~m} \mathrm{ky}^{-1}$ sea-level rise of SL-1 during much of the Holocene. The existence of thick Holocene near-shore sediments, together with $1-2 \mathrm{~km}$ wide marine platforms, however indicates that the area's relative sea level had progressively risen during much of the postglacial period. This led Hsieh et al. (2004) to preferentially consider steeper $\left(>7 \mathrm{~m} \mathrm{ky}^{-1}\right)$ postglacial sea-level curves constrained by the assumed uncertainties of SL-1 for interpretation. This study then clarifies that this part of the sea-level curve is steeper than $7 \mathrm{~m} \mathrm{ky}^{-1}$, and likely to be $8-9 \mathrm{~m} \mathrm{ky}^{-1}$. 


\section{CONCLUSION}

This study is the first application of field data to constrain the 11 - 5 ka eustatic sea-level history in Taiwan, and concludes that: (1) the relatively rapid sea-level rise occurring around the onset of the Holocene (11 - $10 \mathrm{ka}$ ), observed at Barbados, is also evident in Taiwan; (2) the post-glacial sea-level rise (since about $10 \mathrm{ka}$ ) proceeded at an average rate of $8-9 \mathrm{~m} \mathrm{ky}^{-1}$ until approaching the modern level around $6.5-6.6 \mathrm{ka}$, and (3) the sea level rose slowly after 6.5 - 6.6 ka before reaching a climax at $\sim 5 \mathrm{ka}$.

Acknowledgements This research benefits by the projects of Groundwater Monitoring Network Plan in Taiwan and Research of Subsurface Geology of Kaohsiung Metropolitan Area executed by the Central Geological Survey. The authors would like to thank the leaders of the projects, Dr. Tien-Chang Lai, Dr. Chung-Jung Chiang, Dr. Li-Yuan Fei, Dr. Wen-Cheng Chen, Dr. Chih-Cho Huang, and Dr. Jiin-Fa Lee, for their kind support of this study. Gratitude also goes to Miss Su-Ching Chou, Miss Jui-Er Chen, Miss Li-jen Chen, and Mr. Tai-wei Su for their help documenting data during coring processes.

\section{REFERENCES}

Central Geological Survey, 1999: Final Report of Groundwater Monitoring Network in Taiwan - the Hydro-geology Survey in the Choushui Alluvial Fan. Cent. Geol. Surv. MOEA, ROC, 130 pp. (in Chinese).

Central Geological Survey, 2001: Annual Report of Groundwater Monitoring Network in Taiwan (2001) - the Hydro-geological Survey in the Chianan Plain and Lanyang Plain. Cent. Geol. Surv. MOEA, ROC, 212 pp. (in Chinese).

Chen, Y. G., and T. K. Liu, 1996: Sea level changes in the last several thousand years, Penghu Islands, Taiwan Strait. Quat. Res., 45, 254-262.

Chen, Y. G., and T. K. Liu, 2000: Holocene uplift and subsidence along an active tectonic margin, southwestern Taiwan. Quat. Sci. Rev., 19, 923-930.

Fairbanks, R. G., 1989: A 17000-year glacio-eustatic sea level record: influence of glacial melting rates on the Younger Dryas event and deep-ocean circulation. Nature, 342, 637-642.

Hanebuth, T., K. Stattegger, and P. M. Grootes, 2000: Rapid flooding of the Sunda Shelf: a late-glacial sea-level record. Science, 288, 1033-1035.

Hsieh, M. L., P. M. Liew, and M. Y. Hsu, 2004: Holocene tectonic uplift on the Hua-tung coast, eastern Taiwan. Quat. Int., 115-116, 47-70.

Hu, H. T., M. T. Lu, and J. C. Wu, 2001: Annual Report of Subsurface Geological Survey of Kaohsiung Metropolitan Area (2001). Cent. Geol. Surv. MOEA, ROC. (in Chinese).

Huang, S. C., 1991: Some issues about the prehistoric cultures of East Coast. Field Archeol. Taiwan, 2, 1-30 (in Chinese).

Huang, Y. T., 2001: Upper Quaternary Sedimentary Environments and Sequence Stratigraphy of the Tsengwen-his River Basin, Chiannan Plain. Master Thesis., Dept. Geosci., Nat. Taiwan Univ., 187 pp. (in Chinese). 
Lai, T. H., 1995: Late Quaternary Subsurface Geology of the Southern Part of the Choushui Fan-Delta. Master Thesis., Dept. Geosci., Nat. Taiwan Univ., 101 pp. (in Chinese).

Song, S. H., 2001: Sedimentary Sequence Study of Kaohsiung Area Since the Last Glacial Epoch. Master Thesis., Dept. Geosci., Nat. Taiwan Univ., 122 pp. (in Chinese).

Stuiver, M., and P. J. Reimer, 1993: Extended ${ }^{14} \mathrm{C}$ data base and revised CALIB $3.0{ }^{14} \mathrm{C}$ age calibration program. Radiocarbon, 35, 215-230.

Su, F. C., M. T. Lu, W. J. Chi, L. C. Wu, and J. F. Lee, 1999: Report of Subsurface Geological Survey of Kaohsiung Metropolitan Area (1999). Cent. Geol. Surv. MOEA, ROC. (in Chinese).

Sung, W. H., 1969: Changpinian: A newly discovered Preceramic culture from the agglomerate caves on the east coast of Taiwan. Newsletter Chinese Ethnol., 9, 1-27 (in Chinese).

Wu, L. C., 1999: Annual Report of Groundwater Monitoring Network in Taiwan (1999) Deposition Environment and Correlation of Core Sediments in the Chiannan Plain. Cent. Geol. Surv. MOEA, ROC, 119 pp. (in Chinese).

Wu, L. C., 2004: Final Report of Geologic Mapping of the Putzi, Chiali, and Tainan Quadrants in 1: 50,000 Scale. Cent. Geol. Surv. MOEA, ROC, 97 pp. (in Chinese).

Hsieh, M. L., Lai, T. H., Wu, L.C., Lu, W. C., Liu, H.T., and Liew, P. M., 2006: Eustatic sealevel change of $11-5 \mathrm{ka}$ in western Taiwan, constrained by radiocarbon dates of core sediments. Terr. Atmos. Ocean. Sci., 17, 353-370. 